\title{
Analysis of a Standardized Perioperative Pain Management Order Set in Highly Opioid-Tolerant Patients
}

\author{
Alex N. Isaacs, PharmD, BCPS, *† Kellie L. Knight, PharmD, BCPS, $\neq$ and Sarah A. Nisly, PharmD, BCPSł\$
}

\begin{abstract}
Objective: The aim was to assess a standardized order set for perioperative pain management in highly opioid-tolerant patients undergoing elective orthopedic surgery.

Methods: This retrospective chart review evaluated a pain order set in highly opioid-tolerant patients undergoing elective total knee or total hip arthroplasty from January 2010 through August 2012. Based on the date of the surgery, patients were allocated into preimplementation or postimplementation order set groups. The primary outcome assessed whether an adjustment in daily opioid dosage was required within the first 48 hours postoperatively. Secondary outcomes included pain scores, length of hospitalization, and safety outcomes.

Results: Sixty patients were included in the analysis. An adjustment to postoperative opioid therapy occurred in $62 \%$ of the patients in the preimplementation group and in $56 \%$ of postimplementation group patients $(P=0.786)$. There were no differences in median pain scores 48 hours postoperatively $(P=0.348)$. Cumulative toxicity was increased after order set implementation compared with previous patients $(44 \%$ versus $5 \%$, $P<0.005)$; however, opioid doses held for sedation was the only individual toxicity to reach statistical significance $(P=0.011)$.

Conclusions: This study is the first to evaluate a standardized order set for pain management in highly opioid-tolerant patients undergoing elective orthopedic surgery. The order set demonstrated similar efficacy to previous treatment modalities, but opioid-induced sedation was of concern with the order set. After the initial analysis, the order set was modified to minimize opioid-induced sedation. Continual safety analysis is warranted for quality improvement to enhance perioperative pain management in highly opioidtolerant patients.
\end{abstract}

Key Words: order set, pain management, opioid, opioid tolerance, orthopedic surgery

( Patient Saf 2015;00: 00-00)

In 2001, The Joint Commission created analgesic standards to facilitate adequate assessment, treatment, and patient education surrounding pain management. ${ }^{1}$ With the increased use of opioids within the United States, there is a greater risk of developing tolerance as frequent exposure to these medications results in subsequent desensitization to their effects, necessitating higher doses. ${ }^{2,3}$ Opioid tolerance produces many challenges to overcome in the treatment of acute pain, including balancing pain control with adverse drug reactions.

In 2010, there were more than 1 million orthopedic surgical procedures performed in the United States. ${ }^{4}$ In the perioperative

From the *Purdue University College of Pharmacy, West Lafayette; and †Internal Medicine, Eskenazi Health; $₫$ Internal Medicine, Indiana University Health Methodist Hospital; and §Butler University College of Pharmacy and Health Sciences, Indianapolis, Indiana.

Correspondence: Alex N. Isaacs, PharmD, BCPS, Purdue University College of Pharmacy, Eskenazi Health, 640 Eskenazi Ave, Indianapolis, IN 46202 (e-mail: isaacs5@purdue.edu).

The authors disclose no conflict of interest.

Supplemental digital contents are available for this article. Direct URL citations appear in the printed text and are provided in the HTML and PDF versions of this article on the journal's Web site (www.journalpatientsafety.com). Copyright $(2015$ Wolters Kluwer Health, Inc. All rights reserved. setting, effective pain management and use of a standardized pain pathway in orthopedic patients can enhance patient satisfaction scores, reduce length of hospitalization, minimize health care costs, and prevent hospital readmissions and clinic visits. ${ }^{3,5-9}$ However, achieving pain control may be more challenging in opioid-tolerant patients. ${ }^{3,5,7}$ Opioid tolerance has been defined as patients using $60 \mathrm{mg}$ or more of oral morphine equivalents daily for more than 1 week. ${ }^{3,5,10}$ Opioid-tolerant patients often require higher dosages to attain adequate pain control, which must be balanced with the enhanced risk for potential toxicities, including respiratory depression and oversedation. ${ }^{3,5}$ In a study by Oderda et al, ${ }^{11}$ opioidrelated adverse drug events were $75 \%$ more likely to occur in orthopedic surgical patients and $31 \%$ more likely in patients receiving greater than or equal to $10 \mathrm{mg}$ of oral morphine equivalents daily. Orthopedic surgery patients in this study who experienced opioidrelated adverse events had an increased hospital length of stay by 0.52 days and an increase in health care costs by $7.4 \%$.

Despite the opioid epidemic and escalating number of orthopedic surgeries performed annually in the United States, there is limited primary literature on perioperative pain management in highly opioid-tolerant patients, as current recommendations are derived from anecdotal and expert opinions. ${ }^{3,5,6}$ Standardizing order sets are supported by the Institute for Safe Medication Practices (ISMP) to enhance patient care through improved efficacy and safety outcomes. ${ }^{12}$ Although standardized pain management has proven beneficial in orthopedic surgical settings, there is a lack of literature on perioperative pain management in highly opioid-tolerant patients. ${ }^{5-8,10,13-15}$ With the rising prevalence of opioid tolerance, the aim of this research was to retrospectively evaluate the efficacy and safety of a standardized perioperative pain management order set in highly opioid-tolerant patients undergoing elective total knee arthroplasty (TKA) or total hip arthroplasty (THA).

\section{METHODS}

This retrospective chart review evaluated a standardized perioperative pain management order set for orthopedic surgery patients at Indiana University Health Methodist Hospital, an 800-bed, level 1 trauma center located in Indianapolis, IN. Before the order set, there was no standardized treatment plan for the management of acute perioperative pain in orthopedic surgery patients. There was large variability in prescribing with patients receiving scheduled and as-needed oral and intravenous opioids with adjunctive nonopioid medications.

In May 2011, a multidisciplinary team composed of physicians, nurse practitioners, physician assistants, nurses, pharmacists, and other health care professionals developed a perioperative pain management order set to improve postoperative pain scores and enhance patient satisfaction. The order set was first piloted by 1 orthopedic surgeon who performs primarily elective TKA or THA. The order set classifies patients into 1 of 3 different categories based on prescribed daily opioid use before hospital admission (Appendix A, Supplemental Digital Content 1, http://links.lww.com/JPS/A34). Within 30 days before the elective procedure, patients underwent a preoperative clearance visit with an internal medicine physician 
and, as appropriate, were referred to specialists for surgical clearance. In addition, medication histories were obtained at this appointment. At the time of admission, medication histories were again verified by nursing or pharmacy personnel. Any scheduled and as-needed opioid prescriptions used for more than 6 weeks before surgery were included in the quantification of opioid doses to determine the classification of opioid tolerance. A previous internal evaluation illustrated the benefit of the standardized pain management order set, but very few highly opioid-tolerant patients were included in this analysis. As per the derived order set, patients using an average daily dose greater than $90 \mathrm{mg}$ of oral morphine equivalents were categorized as highly opioid tolerant (opioid tolerance level 3).

Treatment options for highly opioid-tolerant patients are illustrated in Appendix A (Supplemental Digital Content 1, http://links.lww.com/JPS/A34). For treatment in the preoperative setting, highly opioid-tolerant patients could receive an oral long-acting opioid the morning before surgery. The postoperative treatment options for highly opioid-tolerant patients included intravenous patient-controlled analgesia, scheduled oral long-acting opioids, scheduled and as-needed short-acting oral medications, and/or as-needed intravenous opioid therapy for breakthrough pain. In addition, providers were encouraged to continue patients on any home pain medications and initiate nonopioid adjuvant therapy, including celecoxib, ketorolac, acetaminophen, and/or pregabalin. The multidisciplinary team developing the order set selected adjuvant therapies based on the literature within arthroplasty ${ }^{16-18}$ and clinical experience with these medications; however, there were additional nonopioid adjunctive therapies not on the order set, which were formulary and available at the discretion of prescribers.

All opioid tolerance level 3 patients undergoing TKA or THA performed by 1 orthopedic surgeon from January 2010 through August 2012 were screened for study inclusion. Patients were eligible for inclusion if they were 18 years or older and hospitalized for more than 48 hours postoperatively. Patients were excluded from the analysis if the incorrect opioid tolerance level was selected based on the preadmission medication history. As the order set was implemented in May 2011, patients were categorized into study groups based on whether the surgery occurred before or after the implementation (Fig. 1).

The primary outcome assessed whether an adjustment in daily opioid dosage was required from the immediate postoperative orders to 48 hours postoperatively. To determine opioid doses, all initially prescribed scheduled and as-needed opioids were converted into oral morphine equivalents to establish the immediate postoperative dose. Daily oral morphine equivalents were calculated at 48 hours after surgery and compared with the immediate postoperative dosages to determine if a change in opioid therapy occurred. If a change occurred, additional analysis evaluated if this was an escalation or deescalation in opioid therapy.

Secondary outcomes were pain scores and hospital length of stay. Pain scores were recorded in the electronic medical record by nurses using a 10-point Likert rating scale with 0 signifying the absence of pain and 10 representing severe pain. The nursing staff recorded pain scores at baseline before surgery, after each opioid administration, and every 2 to 4 hours for the first 24 hours postoperatively, then every 4 to 6 hours thereafter. Baseline pain scores were preoperative assessments of pain within 6 hours before surgery. Median pain scores at baseline and 48 hours postoperatively were analyzed. The hospital length of stay was defined as the number of days admitted at the hospital, including preoperative, operative, and postoperative days. Readmissions after discharge were not evaluated or included within the hospital length of stay.

The primary safety outcome was the cumulative occurrence of respiratory depression, doses held for sedation, and acute kidney injury in both treatment groups. Respiratory depression was defined as a respiratory rate of less than 8 breaths per minute or use of the opioid antagonist, naloxone, for the reversal of respiratory depression. Sedation was defined as scheduled or patientrequested opioid doses, which were held owing to increased patient sedation as noted by a nurse in the electronic medical record. Acute kidney injury was evaluated to determine the safety of adjunctive medications used for pain management. Acute kidney injury was classified using the RIFLE criteria as an increase in serum creatinine greater than 2 times the patient's baseline preoperative laboratory serum creatinine. ${ }^{19}$

Categorical data including the primary outcome and safety data were analyzed using the $\chi^{2}$ test. Nonparametric continuous pain scores for the preimplementation group were compared with those of the postimplementation group using the MannWhitney U-test. Data were analyzed using SPSS version 19. For all statistical analysis, a $P$ value of less than 0.05 was used to define statistical significance.

\section{RESULTS}

There were 498 patients screened for inclusion (Fig. 2). Sixtyfour patients were classified as highly opioid tolerant but 3 were excluded because they were inappropriately classified, and 1 was hospitalized for less than 48 hours postoperatively. A total of 60 patients met inclusion for review, 21 patients in the preimplementation group and 39 patients in the postimplementation group. Baseline characteristics were similar between groups with no statistical differences noted (Table 1). Preadmission daily opioid use was similar between the 2 groups, with median daily dose $180 \mathrm{mg}$ for the preimplementation group and $195 \mathrm{mg}$ for the postimplementation group.

Adjustments to either as-needed or scheduled opioid therapy in the 48-hour postoperative period occurred in 13 patients $(62 \%)$ in the preimplementation group and in 22 patients $(56 \%)$ in the postimplementation group $(P=0.786)$ (Fig. 3). Patients in the preimplementation group had a trend toward escalation of total opioid therapy available ( $52 \%$ versus $28 \%, P=0.065$ ), whereas patients in the postimplementation group trended toward a deescalation of opioid therapy available ( $28 \%$ versus $9 \%, P=0.114)$; however, neither individual adjustment reached statistical significance.

Secondary outcomes are highlighted in Table 2. Median pain scores 48 hours after surgery were 6 of 10 for each group $(P=$ $0.348)$. Moreover, hospital lengths of stay were identical in both groups at 3.33 days $(P=0.926)$. There was increased cumulative toxicity in the postimplementation group (44\% versus $5 \%$, $P<0.005)$. However, the only individual toxicity to reach statistical significance was doses held for sedation $(P<0.011)$.

\section{DISCUSSSION}

With the enhanced use of opioids in the United States, studies are necessary to evaluate the safety and efficacy of perioperative

\begin{tabular}{ccc}
$\begin{array}{c}\text { Pre-Implementation Group } \\
\text { (Jan 2010-Apr 2011) }\end{array}$ & $\begin{array}{c}\text { Order Set Initiation } \\
\text { (May 2011) }\end{array}$ & $\begin{array}{c}\text { Post-Implementation Group } \\
\text { (May 2011-Aug 2012) }\end{array}$ \\
\hline & 0 & 0
\end{tabular}

FIGURE 1. Study timeline. 


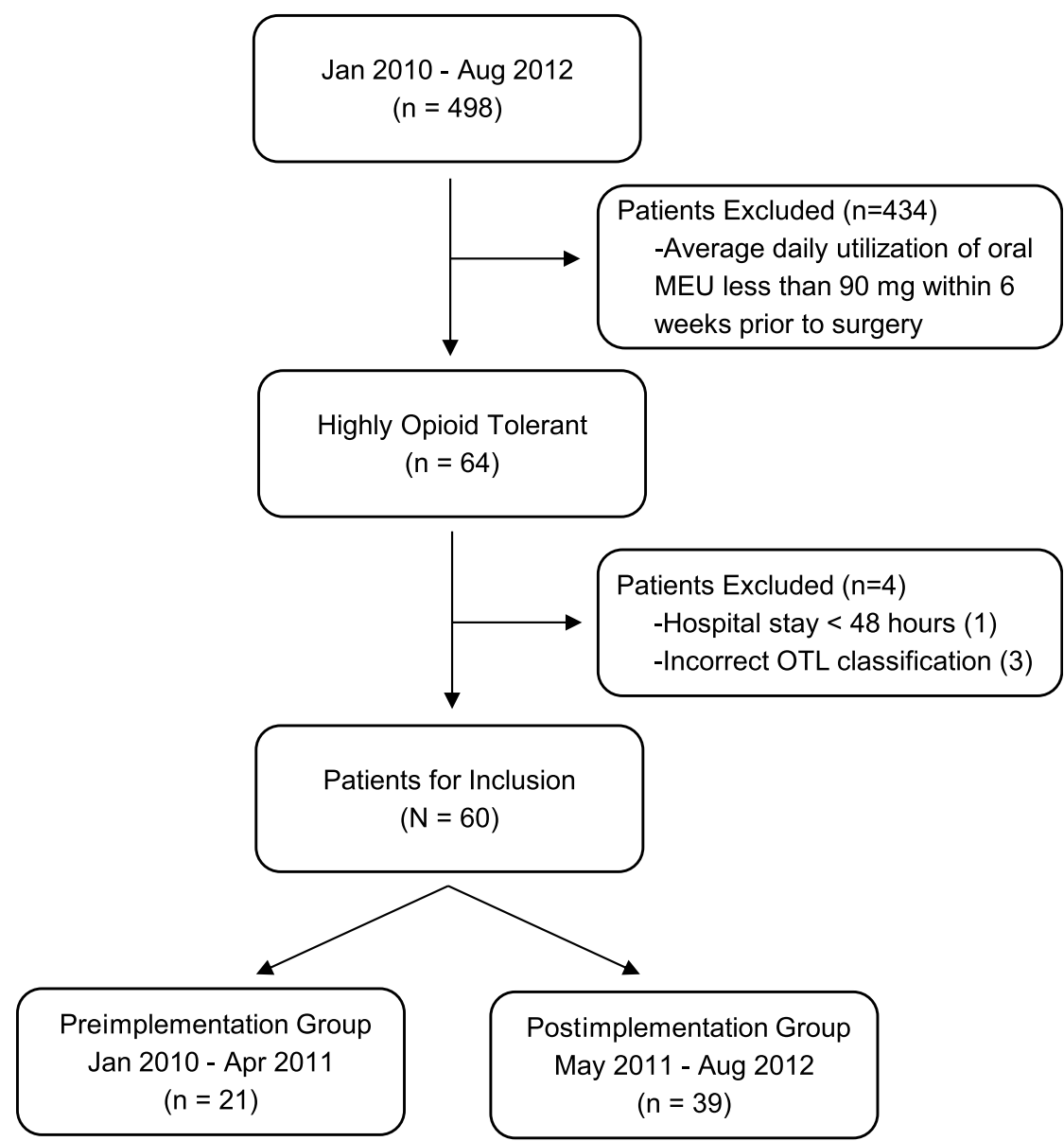

FIGURE 2. Patient extraction for study inclusion. MEU, morphine equivalent units; OTL, opioid tolerance level.

pain management in opioid-tolerant patients. However, there is a void of literature supporting perioperative pain management in this patient population. While not assessing opioid tolerance preoperatively, there is literature supporting the benefits of standardized postoperative pain management within orthopedic surgery. $6,13,14$

A standardized perioperative pain management order set in patients undergoing a THA illustrated a significant decrease in pain scores for the first 4 days postoperatively. Although beneficial, toxicity associated with this treatment strategy was not reported. ${ }^{13}$ In another study, a standardized multimodal analgesic approach in TKA patients resulted in significantly lower pain scores and reduced opioid consumption in the first 48 hours postoperatively. Although there were few differences in toxicities between the groups, nausea and vomiting occurred twice as frequently in the patients being treated with the standardized pain regimen. ${ }^{14}$ Lastly, a study evaluated perioperative outcomes in patients undergoing

TABLE 1. Baseline Characteristics

\begin{tabular}{lcc}
\hline Characteristic & Preimplementation Group $(\mathbf{n}=\mathbf{2 1})$ & Postimplementation Group $(\mathbf{n}=\mathbf{3 9})$ \\
\hline Age, mean (SD), y & $55(14.4)$ & $49(12.5)$ \\
Male, n (\%) & $8(38)$ & $17(44)$ \\
Height, mean (SD), cm & $170(9.1)$ & $163(16.6)$ \\
Weight, mean (SD), kg & $92(30.1)$ & $89(28.9)$ \\
Serum creatinine, median (IQR), mg/dL & $0.80(0.66-1.26)$ & $0.82(0.69-1.02)$ \\
Preadmission daily opioid dose, mg of oral MEU & & $195(120-270)$ \\
Median (IQR) & $180(127-435)$ & $266(242)$ \\
Mean (SD) & $344(337)$ & $21(54)$ \\
Type of orthopedic surgery, n (\%) & & $18(46)$ \\
THA & $13(62)$ & $6(0-9)$ \\
TKA, n (\%) & $8(38)$ &
\end{tabular}

IQR, interquartile range; MEU, morphine equivalent units. 


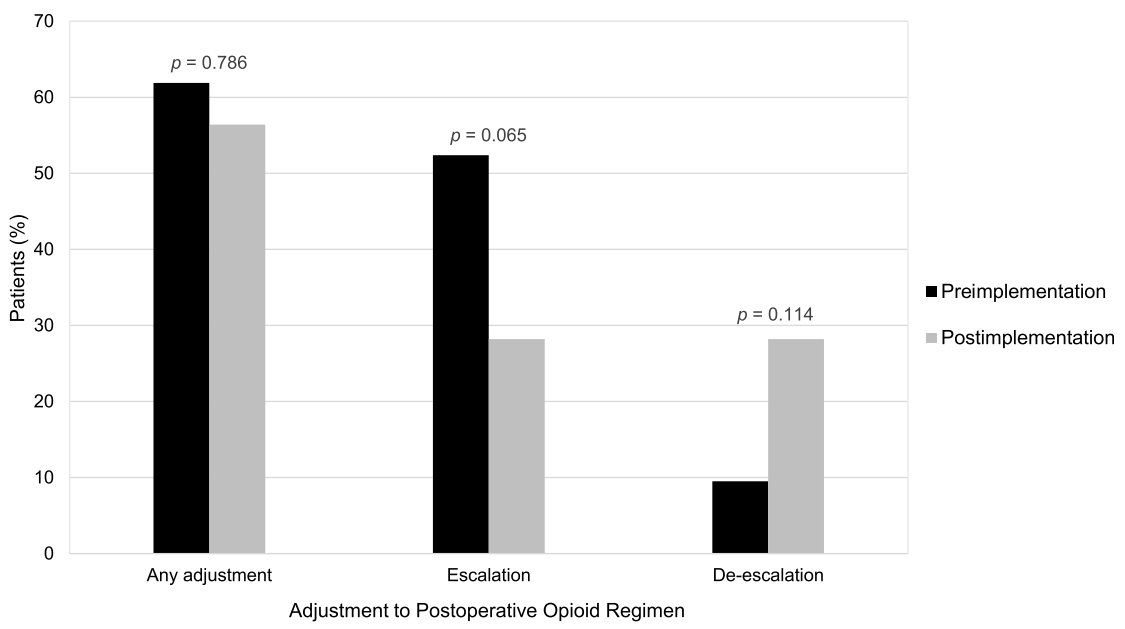

FIGURE 3. Primary outcome.

TKA before and after implementation of a clinical pain pathway. The clinical pain pathway reduced pain scores and opioid consumption in the first 48 hours postoperatively. In addition, there was a significant reduction in hospital length of stay and total direct hospital costs after implementation of this treatment algorithm. ${ }^{6}$ These studies exemplify the potential benefits of a standardized perioperative pain management in orthopedic surgery patients. ${ }^{6,13,14}$ Unfortunately, these studies do not distinguish between opioid use or opioid tolerance before surgery.

The current study is the first to evaluate a standardized treatment approach for opioid-tolerant patients in the perioperative orthopedic setting. The treatment modality resulted in similar pain scores and opioid therapy manipulations between treatment groups. Although there is some concern for increased toxicity in the postimplementation group, the definition of opioid-related adverse effects in this study contributed to the increased incidence of adverse effects compared with the previous literature. In the Oderda retrospective study of 40,368 surgical patients, opioid-related adverse drug effects occurred in 741 patients $(1.8 \%) .{ }^{10}$ A separate analysis of administrative claims data demonstrated opioidrelated adverse drug events occurring in $13.6 \%$ of surgical patients. ${ }^{20}$ Because these studies did not assess sedation, the current study had more frequent adverse effects because of the inclusion of opioid doses held for sedation as an opioid-related adverse effect. Excluding sedation, the other adverse effects were similar between treatment groups in the current study.

Standardized treatment algorithms have been adopted in the inpatient setting for a variety of patient populations to minimize interprescriber variability resulting in enhanced patient safety and outcomes. These order sets are encouraged by the ISMP to reduce variability, enhance workflow, encourage evidencedbased care, and reduce medication errors. ${ }^{12}$ Utility of standardized order sets has demonstrated enhanced efficacy and safety when used within the hospital setting for the management of pneumonia, ${ }^{21}$ sepsis, ${ }^{22,23}$ cirrhosis, ${ }^{24}$ venous thromboembolism prophylaxis, ${ }^{25}$ and patients on warfarin therapy. ${ }^{26}$

Developing safe and effective standardized order sets is a priority of The Joint Commission. ${ }^{27}$ Order sets should be designed to limit selections to appropriate therapeutic options based on patient-specific characteristics. This systematic approach allows providers to receive specific direction, while simultaneously eliminating duplicate choices. ${ }^{27,28}$ If multiple options within a drug class are prescribed, The Joint Commission standards highlight the importance of having clear instructions for use of each duplicate agent. ${ }^{27}$ Explicit instructions enable nursing and other health care providers to deliver safe and effective care as directed by the prescriber. Specifically for opioids, The Joint Commission highlights the importance of safe and judicious prescribing with clear instructions for other health care providers administering medications to help minimize opioid-related adverse effects. ${ }^{28}$ In relation to the current study, the order set was developed with as-needed opioid orders having specific instructions for nursing personnel of when to administer the medications to patients. Clear instructions incorporating the patient pain score and cognitive function help ensure patient safety while assisting in pain control. Irrespective of benefit, standardized order sets require a multidisciplinary team to carefully and thoroughly construct its components. In addition, after implementation of an order set, routine analysis and quality improvement are necessary to ensure optimal patient outcomes.

TABLE 2. Secondary Outcomes

\begin{tabular}{lcrr}
\hline Secondary Outcome & Preimplementation Group (n= 21) & Postimplementation Group (n=39) & $\boldsymbol{P}$ \\
\hline Pain score, median (IQR) & $6(5-8)$ & $6(4-8)$ & 0.568 \\
Hospital length of stay, median (IQR), d & $3.33(3.2-4.2)$ & $3.33(3.0-4.3)$ & 0.926 \\
Cumulative toxicity, n (\%) & $1(5)$ & $17(44)$ & 0.005 \\
Respiratory depression & $1(5)$ & $6(15)$ & 0.404 \\
$\quad$ Naloxone administration & $0(0)$ & $4(10)$ & 0.287 \\
Opioid doses held for sedation, n (\%) & $0(0)$ & $10(26)$ & 0.011 \\
Acute kidney injury, n (\%) & $0(0)$ & $1(3)$ & 1.00 \\
\hline
\end{tabular}

$\mathrm{IQR}$, interquartile range. 
After this initial analysis, opportunities for improvement were identified and implemented to enhance the clinical utility of the standardized perioperative pain management order set. The current study that revealed more opioid doses were held for sedation after the implementation of the order set. Therefore, the order set was modified to eliminate the long-acting and short-acting duplications in scheduled opioid therapy (Appendix B, Supplemental Digital Content 2, http://links.lww.com/JPS/A35). In addition, to minimize the sedative effects of opioid therapy associated with this treatment approach, multidisciplinary education on opioid induced sedation was performed. Since the inclusion period for this analysis, the use of the order set has been expanded and is now available for use within the entire hospital including surgical and medical services. In addition, computerized physician order entry has been implemented, and the order set has been computerized. Lastly, there are efforts underway to implement use of the updated order set within the entire health care system. Continual quality improvement through evaluation and enhancement of the order set will be necessary to optimize pain management in opioid-tolerant patients.

This study does have limitations. The retrospective nature of the study limits data collection, patient randomization, and follow-up assessment. Because of the study design, the patients managed with the order set had to be compared with historical controls, which in itself has inherent limitations. The small sample size makes it difficult to extract to all opioid-tolerant orthopedic patients. Although limiting the analysis to 1 surgeon is beneficial for reduction in prescribing variability, this may have prevented adjustment in opioid therapy compared with studies with multiple providers. Moreover, evaluating each patient's opioid tolerance was heavily dependent on accurate medication histories performed at the preoperative visit. Finally, patients within the highly opioid tolerance group had large variability in home opioid use, making it difficult to control for variability between groups. Despite the limitations, this remains the first study evaluating the impact of the standardization of perioperative pain management in highly opioid-tolerant orthopedic surgery patients.

\section{CONCLUSIONS}

Standardized order sets are an organized approach to safe and effective perioperative pain management as supported by The Joint Commission and ISMP. This study is the first to evaluate a standardized order set for pain management in highly opioidtolerant patients undergoing elective orthopedic surgery. The order set demonstrated similar efficacy to previous treatment modalities, but opioid-induced sedation was of concern after implementation of the standardized order set. After the initial analysis, a modification to the order set was implemented to minimize the risk of opioid-induced sedation. As with any new order set, continual safety analysis is warranted for quality improvement to enhance perioperative pain management in highly opioidtolerant patients.

\section{ACKNOWLEDGMENTS}

The authors would like to acknowledge the following team members for contributing to the creation of the opioid tolerance order set: Christina Bortone, NP; Brian McCrate, PharmD; Patrick McQuillan, MD; Jill Payne, MSN; Lara Pesavento, MD; J. Andrew Parr, MD; Meggie Ruch, PA-C; Jim Ryser, MA; Kristen Swartzell, MSN; and Julie Williams, PharmD.

\section{REFERENCES}

1. The Joint Commission. Facts about pain management. The Joint Commission Web site. February 4, 2014. Available at: http://www. jointcommission.org/pain_management/. Accessed November 5, 2014.
2. Manchikanti L, Helm S 2nd, Fellows B, et al. Opioid epidemic in the United States. Pain Physician. 2012;15(suppl 3):ES9-ES38.

3. Mitra S, Sinatra RS. Perioperative management of acute pain in the opioid-dependent patient. Anesthesiology. 2004;101:212-227.

4. Faststats - Inpatient Surgery. The Centers for Disease Control and Prevention Web site. May 14, 2014. Available at: http://www.cdc.gov/nchs/ fastats/inpatient-surgery.htm. Accessed November 5, 2014.

5. Gordon D, Inturrisi CE, Greensmith JE, et al. Perioperative pain management in the opioid-tolerant individual. J Pain. 2008;9:383-387.

6. Duncan CM, Moeschler SM, Horlocker TT, et al. A self-paired comparison of perioperative outcomes before and after implementation of a clinical pathway in patients undergoing total knee arthroplasty. Reg Anesth Pain Med. 2013;38:533-538.

7. Patanwala AE, Jarzyna DL, Miller MD, et al. Comparison of opioid requirements and analgesic response in opioid-tolerant versus opioid-naïve patients after total knee arthroplasty. Pharmacotherapy. 2008;28: 1453-1460.

8. Ayalon O, Liu S, Flics S, et al. A multimodal clinical pathway can reduce length of stay after total knee arthroplasty. HSS J. 2011;7:9-15.

9. Oderda G. Challenges in the management of acute postsurgical pain. Pharmacotherapy. 2012;32(Suppl 9):6S-11S.

10. Kopf A, Banzhaf A, Stein C. Perioperative management of the chronic pain patient. Best Pract Res Clin Anaesthesiol. 2005;19:59-76.

11. Oderda GM, Said Q, Evans RS, et al. Opioid-related adverse drug events in surgical hospitalizations: impact on costs and length of stay. Ann Pharmacother. 2007;41:400-407.

12. The Institute for Safe Medication Practices (ISMP) guidelines for standard order sets ISMP Web site. November 5, 2014. Available at: http://www.ismp.org/tools/guidelines/standardordersets.pdf. Accessed September 25, 2014.

13. Lee KJ, Min BW, Cho CH, et al. Multimodal approach to postoperative pain control in patients undergoing rotator cuff repair. Clin Orthop Surg. 2009; 1:155-160.

14. Fu PL, Xiao J, Zhu YL, et al. Efficacy of a multimodal analgesia protocol in total knee arthroplasty: a randomized, controlled trial. J Int Med Res. 2010;38:1404-1412.

15. Huxtable CA, Roberts LJ, Somogyi AA, et al. Acute pain management in opioid-tolerant patients: a growing challenge. Anaesth Intensive Care. 2011;39:804-823.

16. Clarke H, Pereira S, Kennedy D, et al. Gabapentin decreases morphine consumption and improves functional recovery following total knee arthroplasty. Pain Res Manag. 2009;14:217-222.

17. Post ZD, Restrepo C, Kahl LK, et al. A prospective evaluation of 2 different pain management protocols for total hip arthroplasty. J Arthroplasty. 2010;25:410-415.

18. Buvanendran A, Kroin JS, Della Valle CJ, et al. Perioperative oral pregabalin reduces chronic pain after total knee arthroplasty: a prospective, randomized, controlled trial. Anesth Analg. 2010;110:199-207.

19. Bellomo R, Ronco C, Kellum JA, et al. Acute renal failure-definition, outcome measures, animal models, fluid therapy and information technology needs: the Second International Consensus Conference of the Acute Dialysis Quality Initiative (ADQI) Group. Crit Care. 2004;8: R204-R212.

20. Kessler ER, Shah M, Gruschkus SK, et al. Cost and quality implications of opioid-based postsurgical pain control using administrative claims data from a large health system: opioid-related adverse events and their impact on clinical and economic outcomes. Pharmacotherapy. 2013;33: 383-391.

21. Fleming NS, Ogola G, Ballard DJ. Implementing a standardized order set for community-acquired pneumonia: impact on mortality and cost. Jt Comm J Qual Patient Saf. 2009;35:414-421. 
22. Rivers EP, Coba V, Rudis M. Standardized order sets for the treatment of severe sepsis and septic shock. Expert Rev Anti Infect Ther. 2009;7:1075-1079.

23. Thiel SW, Asghar MF, Micek ST, et al. Hospital-wide impact of a standardized order set for the management of bacteremic severe sepsis. Crit Care Med. 2009;37:819-824.

24. Kijsirichareanchai K, Ngamruengphong S, Rakvit A, et al. The utilization of standardized order sets using AASLD guidelines for patients with suspected cirrhosis and acute gastrointestinal bleeding. Qual Manag Health Care. 2013;22:146-151.

25. Khanna R, Vittinghoff E, Maselli J, et al. Unintended consequences of a standard admission order set on venous thromboembolism prophylaxis and patient outcomes. J Gen Intern Med. 2012;27: 318-324.

26. Nisly S, Shiltz ED, VanArsdale V, et al. Implementation of an order set to adhere to national patient safety goals for warfarin therapy. Hosp Pharm. 2013;48:828-832.

27. The Joint Commission. 2015 Comprehensive Accreditation Manual for Hospitals. Oak Brook, IL: Joint Commission Resources; 2014.

28. The Joint Commission. Safe use of opioids in hospitals. The Joint Commission Web site. August 8, 2012. Available at: http://www. jointcommission.org/assets/1/18/SEA_49_opioids_8_2_12_final.pdf. Accessed November 5, 2014. 